\title{
Improving self-regulation in adolescents: current evidence for the role of mindfulness-based cognitive therapy
}

This article was published in the following Dove Press journal:

Adolescent Health, Medicine and Therapeutics

13 September 2016

Number of times this article has been viewed

\author{
Carisa Perry-Parrish' \\ Nikeea Copeland-Linder ${ }^{2}$ \\ Lindsey Webb ${ }^{2}$ \\ Ashley H Shields' \\ Erica MS Sibinga ${ }^{2}$ \\ 'Department of Psychiatry and \\ Behavioral Sciences, Division of \\ Child and Adolescent Psychiatry, \\ Pediatric Medical Psychology Program, \\ ${ }^{2}$ Department of Pediatrics and \\ Adolescent Medicine, Johns Hopkins \\ School of Medicine, Baltimore, MD, \\ USA
}

\begin{abstract}
Mindfulness-based cognitive therapy (MBCT) was introduced in 1995 to address the problem of recurrent depression. MBCT is based on the notion that meditation helps individuals effectively deploy and regulate attention to effectively manage and treat a range of psychological symptoms, including emotional responses to stress, anxiety, and depression. Several studies demonstrate that mindfulness approaches can effectively reduce negative emotional reactions that result from and/or exacerbate psychiatric difficulties and exposure to stressors among children, adolescents, and their parents. Mindfulness may be particularly relevant for youth with maladaptive cognitive processes such as rumination. Clinical experience regarding the utility of mindfulness-based approaches, including MBCT, is being increasingly supported by empirical studies to optimize the effective treatment of youth with a range of challenging symptoms. This paper provides a description of MBCT, including mindfulness practices, theoretical mechanisms of action, and targeted review of studies in adolescents.
\end{abstract}

Keywords: youth, mindfulness-based cognitive therapy, adolescents

\section{Introduction}

Many youths are at risk for experiencing stressors during adolescence that may lead to maladaptive coping strategies to manage negative affective experiences. Adolescence is a period of development and change for youth across multiple domains. ${ }^{1}$ Although viewed by many as representing a dark and stormy period of emotional turbulence, most adolescents do not exhibit this pattern. However, epidemiological studies identify the adolescent years as conferring risk for internalizing problems of depression and anxiety for a minority of youth, ${ }^{1,2}$ as well as introducing the increased potential for sensation seeking and experimentation compared to younger children. Peers take on new social salience as teens increasingly reflect on their status in the interpersonal landscape, and social contextual consideration likely influences adolescents' decisions when in the company of other youth. The interest in exciting emotional experiences and the affordances of social standing bring youth into contact with new types of stressors not previously experienced during childhood. While emotion regulation and coping may protect youth by mitigating the negative effects of stressors, ${ }^{1,3,4}$ chronic exposure to stressors may deplete their coping resources. Significant, recurrent, and/ or ongoing stress may contribute to toxic stress, in which an individual's ability to manage or cope with stress is overwhelmed. ${ }^{5}$ Increasingly, there are calls for broader thinking about how to mitigate the maladaptive aspects of stress experienced in the teen years to reduce the risk for long-term difficulties. Mindfulness-based treatments
Correspondence: Carisa Perry-Parrish Department of Psychiatry and Behavioral Sciences, Division of Child and Adolescent Psychiatry, Pediatric Medica Psychology Program, Johns Hopkins School of Medicine, 1800 Orleans St, Bloomberg Children's Center I2N, Baltimore, MD 21 287, USA

Tel + I 4I0955 7674

Email cparris5@jhmi.edu 
may provide a beneficial approach to help many adolescents reduce stress and other psychological problems. In this paper, we will review the history of mindfulness-based cognitive therapy (MBCT), literature pertaining to stress and coping experienced among youth, and evidence of its effectiveness with adolescents.

\section{History of MBCT}

Mindfulness has been defined as "paying attention in a particular way: on purpose, in the present moment, and nonjudgmentally". ${ }^{6}$ Mindfulness instruction is intended to enhance awareness of and attunement to what is happening internally and externally with friendly curiosity and without judgment. The nonjudgmental awareness that is enhanced in mindfulness interventions is theorized to facilitate self-regulatory processes and coping, particularly during stressful experiences. ${ }^{7}$ Therefore, mindful interventions may be helpful in alleviating suffering among youth dealing with life stress.

Mindfulness instruction includes exercises and techniques that cultivate an intentional focus on present-moment experience without being self-critical or judgmental. The goal of this training ${ }^{6,7}$ is to help individuals accept unpleasant and painful experiences without attempting to change the experience. ${ }^{8}$ However, reducing or eliminating pain and discomfort is an understandable and natural tendency, so many mindfulness-based programs adopt a position of balancing the desire for change with an acceptance of the reality of uncomfortable experiences. ${ }^{8}$

The use of mindfulness meditation practices to reduce distress has been a feature of many Eastern philosophical traditions (eg, Buddhism) for hundreds of years, ${ }^{9}$ and have been increasingly used in Western medicine for well over 30 years. ${ }^{10-12}$ Meditation practices emerged from a body of Buddhist and other contemplative traditions that represent a complementary approach that joins psychological approaches to reduce stress and discomfort, ${ }^{13}$ including cognitive-behavioral therapies and relaxation techniques. These approaches emphasize the importance of mindful acceptance and behavioral change as core processes to ameliorate mental and physical suffering. Mindfulness-based stress reduction (MBSR) was developed for use in adults suffering with chronic pain and ongoing stressors. ${ }^{9-11}$ Since the establishment of MBSR in 1979, several mindfulness-based interventions have been implemented to address a variety of psychological problems among adults, ${ }^{12}$ including MBCT, ${ }^{13}$ dialectical behavior therapy, ${ }^{14}$ acceptance and commitment therapy, ${ }^{15}$ and mindfulness-based relapse prevention. ${ }^{16}$
MBSR was developed at the University of Massachusetts Medical Center and is an 8-10 week program consisting of $2 \frac{1}{2}$ hour sessions per week. The intervention is group-based, and participants are expected to commit to regular daily practice during program participation. ${ }^{9}$ The program content focuses on mindfulness meditation practice, self-awareness of the body, yoga, and addresses barriers to the use of mindfulness practices during stressful moments.

Since the introduction of MBSR in 1979, several other psychological treatments emphasizing mindfulness and mindful acceptance have emerged within the behavioral and cognitive-behavioral movement. A common theme among these approaches was a new focus on affect and emotional experiences as a primary target for treatment. For example, dialectical behavior therapy incorporates mindfulness and acceptance practices to address severe emotional dysregulation. ${ }^{14}$ Acceptance and commitment therapy focuses on the importance of the function and context of experiential avoidance to improve psychological flexibility. ${ }^{17} \mathrm{MBCT}{ }^{18}$ modified cognitive therapy to incorporate mindfulness techniques with the express purposes of training strategic attention deployment, particularly among adults with recurrent depression. Mindfulness-based approaches are distinguished from other cognitive-behavioral treatments in that cognitive behavioral therapy approaches focus on changing one's thoughts and behavior while mindfulnessbased approaches focus on balancing desire for changed experiences with acceptance of the present moment.

The format of MBCT mirrors the practices and approach of MBSR. The core MBSR curriculum forms the basis of MBCT, including formal exercises (eg, the raisin exercise, body scan, sitting meditation) and informal meditation in daily life (eg, washing dishes, brushing one's teeth, taking out the trash). MBCT for individuals with a history of depression focuses the didactic portion of the course on depression specifically, rather than stress more generally. A key similarity is the emphasis on experiential exercises. Both MBSR and MBCT emphasize that instructors must have their own mindfulness practice and ongoing professional development to maintain adequate practice in order to support the accurate teaching of mindfulness exercises to others; both approaches also emphasize the importance of participant practice, both in group and at home, to facilitate the psychological changes theoretically associated with mindfulness training. From the cognitive perspective of MBCT, home-based meditation sessions are important not only to reinforce habits, but also to acquire new memories of nondepressogenic coping schemas. ${ }^{18}$ Regardless of the actual mechanism, ongoing 
mindfulness practice ensures familiarity with the techniques that are presumed to improve self-regulation, likely through a variety of channels.

MBCT initially emerged as an effort to reduce the risk for depressive episodes by targeting maladaptive mood regulation. In the two decades since its introduction, MBCT has been extended to multiple applications for adults, including generalized anxiety disorder, treatment-resistant depressive disorder, cancer, suicidal behavior, and bipolar disorder. ${ }^{19}$ Most of the research has been conducted with adult samples, with a much smaller research base with adolescents. We will next review the role of stress in adolescence, potential mechanisms of mindfulness in MBCT, and then finally review the evidence for MBCT in adolescents.

\section{Review of literature Stress exposure in youth}

Stressors experienced in childhood and adolescence have been associated with maladaptive outcomes, including internalizing problems, externalizing behaviors, academic difficulties, and health risk behaviors. ${ }^{20,21}$ Exposure to some stressors increases as youth age into adolescence. ${ }^{22}$ This picture is compounded with concern for minority youth, particularly those living in low-resource neighborhoods, who encounter a disproportionate share of stressors due to structural inequality. ${ }^{23,24}$ Minority youth are more likely to experience the death of a family member or friend, to have a relative with negative legal encounters (eg, arrested or jailed), to have to become a caretaker, and to be placed in the foster care system. ${ }^{24,25}$ Nevertheless, the effects of stress on coping and psychological functioning are important for all youth, as recurrent stressors may continuously tax an adolescent's native self-regulatory system. As recent work has shown that coping can temper the effects of stress on development of depressive symptoms over time in youth, ${ }^{24,26}$ interventions that enhance adolescents' abilities to cope effectively with inevitable stress may provide a protective effect against psychological difficulties.

Exposure to stressors may increase the risk for developing emotional and behavioral problems, particularly if youth rely on maladaptive coping and emotion regulation strategies. Many stressful circumstances are difficult to change, and negative thoughts associated with these stressors may be quite valid and accurately reflect genuinely negative experiences. Rumination is a process that has been implicated in depressive disorders, ${ }^{27}$ and has been shown to increase the risk for recurrence and may respond favorably to $\mathrm{MBCT} .^{28}$ Mindfulness-based interventions target the emotional and attentional processes associated with stress and may support the development of adolescents' self-regulation. In the next section, we will review theoretical models that further detail how mindfulness-based treatments may reduce the negative effects of stress and highlight empirically evaluated mindfulness treatments for adolescents.

\section{Effects of mindfulness on self-regulation}

Mindfulness has been broadly theorized to improve selfregulation of emotions, behavior, and cognitive processes. ${ }^{29}$ Emotion regulation is regarded as a fundamental aspect of many kinds of youth psychopathology generally ${ }^{30}$ and a potential mediator of the relationship between exposure to risk and healthy developmental outcomes for minority youth. ${ }^{5}$ Self-regulation of negative emotions, ${ }^{31}$ such as anger and sadness, is related to social and peer acceptance across childhood and adolescence; thus, in addition to psychological benefits, mindfulness approaches may improve emotion regulation which may, in turn, enhance social development. ${ }^{32-34}$ The psychological shift theorized to occur with mindfulness may contribute to improvements in associated cognitive, emotional, social, and behavioral domains (see Table 1 for examples). Cognitive information-processing models have been useful in explaining mindfulness mechanisms. In one of their earliest papers introducing the theoretical foundation for MBCT, Teasdale et al explain that "“central engine resources' are devoted to repetitive, 'ruminative,' information processing cycles motivated to the attainment of central personal goals, or intentions, that can be neither attained nor relinquished". ${ }^{18}$ The inability to strategically direct one's attention denotes a critical element of one's cognitive processing resources and is theoretically constrained here by emotional considerations of attachment to a particular outcome in a complex matrix of individual goals and desires. We have previously discussed ${ }^{7}$ the multiple ways by which an information-processing model ${ }^{18,35}$ might explain how mindfulness works to modify attentional and emotional self-regulation processes: 1) interrupting automatic, "mindless" habits and cognitive scripts associated with maladaptive behavior; 2) changing an

Table I Potential psychological changes associated with mindfulness training

Improved intentional shifts in attention

Increased flexibility of attention

Reduced emotional intensity/duration and secondary emotional responses Reduced belief in automatic thoughts

Enhanced ability for learning

Enhanced treatment motivation

Note: Data from Perry-Parrish et al. ${ }^{7}$ 
individual's relationship to his or her own memory activation (eg, neutrally observing a memory, rather than attempting to inhibit it, or reacting in an emotionally negative way); 3) becoming desensitized to previous emotional triggers for behavior; and 4) developing increased attention to and awareness of one's own general cognitive and emotional processes. Therefore, mindfulness "may change automatic response tendencies when the patient observes, describes, and participates in emotional experiences without acting on them". ${ }^{36}$ Likewise, mindfulness may help reduce ineffective action tendencies that are linked with emotion dysregulation, ${ }^{36}$ and the reduction of inflexibility and avoidance may allow individuals to observe their psychological experiences instead of attempting to control them. ${ }^{37}$

Mindfulness approaches are theorized to result in improved self-regulation that emerges from increased acceptance and self-awareness, such as noticing unpleasant emotions and distress as experiences that can be accepted, rather than impulsively reacted to, ruminated over, or chronically avoided in an ineffective manner. ${ }^{7,13,37,38}$ An enhanced acceptance of internal experiences may decrease suffering and distress in response to stress. Mindfulness-based treatment may decrease symptoms through exposure to emotional and psychological stimuli, reduce maladaptive cognitive processes that inadvertently maintain negative mood states, change attitude/cognitive stance, and increase self-regulation and coping skills that promote acceptance. ${ }^{13}$ Therefore, mindfulness may address maladaptive self-soothing and avoidant responses which function to maintain homeostasis that may accompany many forms of psychological disorders, including anxiety, depression, inattention, and other forms of psychopathology.

In summary, mindfulness approaches emphasize approaching and accepting one's experiences, rather than the chronic efforts in avoiding uncomfortable or undesired experiences. ${ }^{15}$ Our theoretical model (Figure 1) reflects the range of potential changes associated with mindfulness instruction, including the intertwined self-regulatory processes of improved coping, positive cognitive changes, and improved psychological functioning.

\section{Mindfulness-based therapies: empirical evidence}

Studies of mindfulness-based interventions for use with youth and adolescents are still emerging. This growing literature provides preliminary evidence in support of the feasibility and benefits of mindfulness-based treatments for use in pediatric populations ${ }^{39-41}$ and well-accepted by youth. ${ }^{42,43}$ Several mindfulness programs, including MBCT, have been adapted for use with youth. Adaptations include shorter practice periods when introducing formal mindfulness techniques and gradual increase in duration as the course progresses; making language appropriate for youth; and selecting ageappropriate mindfulness activities. ${ }^{44}$ Our review of MBCT among adolescents will focus on identifying the effects and then suggesting areas for ongoing research.

In contrast to the adult literature, there are fewer empirical articles evaluating the effects of mindfulness training for

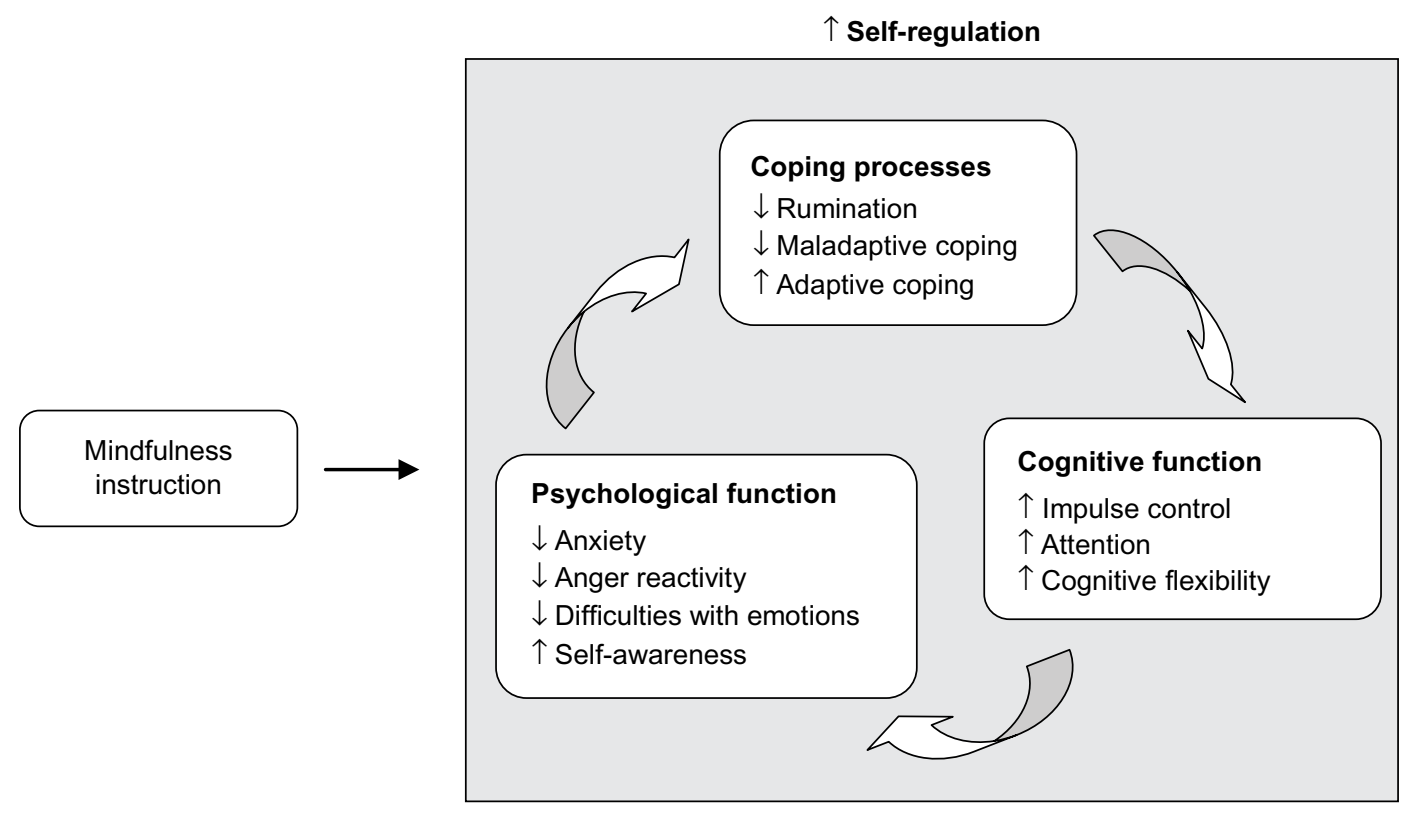

Figure I Mindfulness instruction and improved self-regulation. Note: Data from Perry-Parrish et al. ${ }^{7}$ 
youth. However, this growing body of knowledge has provided encouraging preliminary results regarding acceptability and feasibility in pediatric samples, suggesting that there is a continued need for more rigorous research on mindfulness program effects. ${ }^{45}$ Moreover, several of these studies document positive changes in response to these mindfulness interventions. For example, mindfulness instruction in high school students led to improved blood pressure. ${ }^{46}$ A recent randomized controlled trial (RCT) comparing mindfulness with hatha yoga and waitlist control showed that only mindfulness led to a significant improvement in working memory capacity. ${ }^{47}$ Another RCT of disordered eating prevention for adolescent girls showed benefit when mindfulness was delivered in optimal facilitation, ${ }^{48}$ suggesting that program delivery elements are important considerations of potential effect. Given the importance of MBSR in the original development of MBCT, we will review existing studies on MBSR in adolescents as well as MBCT.

Several studies have examined MBSR adapted for youth, showing feasibility, acceptability, and positive effects. Studies of MBSR from our program in an urban outpatient primary care clinic have demonstrated acceptability, feasibility, and potential benefit related to improved relationships and coping, and reduced conflict engagement, anxiety, and stress. ${ }^{44,49,50}$ Furthermore, a small RCT of MBSR for urban youth aged 13-21 recruited from a primary care pediatric clinic demonstrated that MBSR could be feasibly and acceptably adapted for urban youth. ${ }^{42}$ Qualitative data suggested that youth from the adapted MBSR program perceived improved self-regulation following instruction in mindfulness, including increased feelings of calm, self-awareness, and conflict avoidance, compared with youth in the active control program. Another RCT of MBSR compared with an active control program focused on boys in an urban school setting and documented decreased anxiety and rumination, with a trend for reduced negative coping and possible attenuation of cortisol response over the academic year. ${ }^{43}$ An RCT of MBSR compared with an active control program (health education) for human immunodeficiency virus-positive, predominantly African-American youth found improvements in both self-regulation and physiological outcomes, with MBSR participants being more likely to have lower human immunodeficiency virus viral loads at 4-6 months follow-up compared to the control group. ${ }^{51}$ Although these studies are small, they demonstrate that mindfulness-based therapies can be successfully adapted for use with youth and have a positive effect compared with an active control program. A larger RCT compared a school-based MBSR program for primary prevention with an active control program in two urban public schools $(\mathrm{N}=300)$. This trial showed that MBSR participants had statistically significant reductions in symptoms of depression, negative coping, negative affect, somatization, self-hostility, and posttraumatic stress symptoms. ${ }^{52}$ Other trials of MBSR are promising as well. MBSR was compared with usual care for adolescents in outpatient psychiatric treatment using an RCT design and showed significantly reduced anxiety and depression and improvements in global psychiatric functioning. ${ }^{53}$ In a treatment study of adolescent substance use, ${ }^{54}$ MBSR was chosen as an effective complement to other therapeutic components (ie, sleep hygiene, stimulus control, and cognitive therapy) in reducing sleep problems and was well accepted. Taken together, these results are promising; yet, all of these studies underscore the need for rigorous study designs to enhance the methodological evaluation of mindfulness instruction for youth.

Fewer studies address the effects of MBCT programs for adolescents. MBCT has been modified for children as a downward extension of manualized MBCT. ${ }^{55}$ Adaptations were based on developmental considerations to optimize the acceptability and feasibility of the intervention with youth. Key adaptations include shorter, more repetitious sessions (eg, 90 minutes instead of 2 hours, delivered over 12 weeks instead of 8 weeks); smaller groups (eg, six to eight students to two instructors, versus 30 adult participants in a group); use of formal limit-setting to prevent misbehavior (eg, review of rules); use of multisensory mindfulness experiences to address the cognitive capacity of this age group; use of incentives for session attendance and completion of homework (eg, stickers); and family involvement (eg, having parents support a child's home practice, and encouraging mindful speech and behaviors at home). A small feasibility trial of MBCT adapted for children (MBCT-C) showed good acceptability and reduced internalizing (eg, anxiety, depression) and externalizing symptoms (eg, disruptive behavior) among a community sample of pre- and early adolescents, aged 9-12 years, with a history of academic problems. ${ }^{55}$ Another trial of MBCT-C examined youth 9-13 years old and found reductions in attention problems. ${ }^{56}$ This group had been referred for reading difficulties ( $84 \%$ ethnic minority); students were randomized to MBCT-C by age cohort (ie, 9-10 year olds or 11-13 year olds) or to a waitlist prior to participating in a future MBCT-C group. In addition, a strong association between attention problems and behavior problems was noted and the authors speculated that MBCT$\mathrm{C}$ could help promote improved behavioral functioning by reducing inattention. ${ }^{56}$ 
MBCT has been investigated in a group of adolescents with externalizing behavior problems in the Netherlands; ${ }^{57}$ youth had diagnoses of attention deficit/hyperactivity disorder (ADHD), oppositional defiant disorder or conduct disorder, or autism spectrum disorder. This adaptation for adolescents and their parents was based on the original MBCT manual and used the same sequence of mindfulness activities (eg, body scan, mindful breathing, breathing space). Adolescents $(\mathrm{N}=14)$ and parents attended separate groups for 1.5 hours simultaneously; the group of adolescents focused on their difficulties, and the group of parents emphasized their role as a parent, including acceptance of their adolescent child, difficulties in communication, and family interactions. Attendance to group sessions was incentivized; adolescent participants earned points that could be exchanged for material and social rewards from parents, and they could earn money for completing the course. Postgroup analyses indicated improvement in adolescent attention, self-control, and mindful awareness; adolescents also reported decreased externalizing symptoms and social problems.

A more recently published study of MBCT in youth examined adolescents with ADHD in Canada. ${ }^{58}$ Adolescents were $13-18$ years old and $78 \%$ had comorbid psychiatric disorders. Teens and their parents $(\mathrm{N}=18)$ attended separate weekly sessions of 90 minutes for 8 weeks. The MYmind program is based on $\mathrm{MBCT}^{12}$ and was developed by Bogels and colleagues in the Netherlands. Didactic content focused on mindfulness instruction as a useful tool for coping with ADHD symptoms, stress, family interactions, and difficult emotions. Results indicated reduced inattention, conduct problems, and peer problems following MBCT. Parents also reported reduced parenting stress and increased mindful parenting. In light of the good retention shown in this study, as well as the encouraging results, the MYmind curriculum is a promising adaptation of MBCT for adolescents with ADHD and other externalizing disorders.

Finally, one study has examined MBCT for adolescents with depression. ${ }^{59}$ In this study, adolescents $(\mathrm{N}=11)$ aged 12-18 years with residual symptoms of depression following treatment were recruited to receive adapted MBCT. The MBCT approach was based on manualized adult MBCT as well as the modified MBCT-C adaptation; there were 8 weekly groups, and adolescent participants indicated high levels of satisfaction with the group intervention. Attendance rates were good, with $64 \%$ completing the course. Nearly all the participants were female. The small sample size limited analyses of potential treatment effects, but there were large effect sizes observed for changes in depressive symptoms and impact of symptoms, and more modest decreases in worry and rumination, and in improved quality of life. Thus, paralleling the extensive research base on MBCT for adults with a history of depression, this preliminary investigation would suggest that mindfulness instruction for adolescents could be a promising approach to reduce depressive symptoms.

In summary, evidence suggests that MBCT may be beneficial for adolescents and their parents to enhance selfregulation and coping. The results are encouraging, but the small sample sizes and lack of rigorous designs call for further research to clarify the extent and magnitude of the potential benefit for other groups of adolescents. Existing shortcomings of the studies examined here include: low number of participants; lack of a control group; limited follow-up period; and variable populations examined, including those without a formal psychiatric diagnosis. There is not currently a standard adolescent MBCT adaptation, which may make it difficult to compare across trials of various versions of modified MBCT with adolescents.

\section{Recommendations for future research and clinical care}

Mindfulness-based treatment approaches have been shown to improve overall well-being. ${ }^{7}$ Mindfulness may enhance selfregulation and the reciprocal processes of cognitive functioning (eg, attentional deployment), psychological functioning (eg, emotional states), and coping (eg, responses to emotional challenge) (Figure 1). Mindfulness-based approaches reduce psychological symptoms, improve emotion regulation, improve attention and the ability to focus, and reduce maladaptive coping and rumination. ${ }^{7}$ These improvements are further associated with increased calmness, improved relationships, and reduced stress. While there is a great enthusiasm among many who study mindfulness instruction for youth and optimism for the benefits that mindfulness practices may yield, there is still a need for rigorous scientific evaluation of mindfulness interventions for adolescents.

Future research on mindfulness-based interventions for adolescents should prioritize improving the methods for evaluating mindfulness instructions to ensure that children and youth have access to optimal clinical care that is evidence based. MBCT has demonstrated a wealth of benefit among adults, but has not been tested as frequently or as rigorously among youth. Mindfulness interventions, including MBCT, need to be evidence based, and dissemination of these interventions needs to ensure fidelity. As with other efforts to disseminate treatments that have demonstrated efficacy, it will be crucial to determine how MBCT can survive the 
transfer from adults to adolescents, and then from optimal delivery in rigorous studies to more typical settings of care, such as community mental health centers. The application of implementation science to efforts to disseminate mindfulness instruction by MBCT will play a critical role in the success of delivering high-quality mindfulness programming. Second, future research needs to continue to identify the potential mechanisms of change associated with mindfulness instruction. When MBCT demonstrates positive changes for youth, it will continue to be important to identify which emotional and psychological processes are changed (eg, attentional processes, rumination, reappraisal) and how they are improved.

Mindfulness techniques represent a group of complementary treatments that are beneficial for youth presenting with a range of behavioral, emotional, and somatic symptoms, as mindfulness instructions change the relationship to one's experiences in a positive way. Encouraging mindful awareness of one's present-moment experiences, as well as associated thoughts and emotions, allows an individual to recognize both the sensation and responses to the stimulus. As a result, individuals decrease attachment to the associated thoughts and emotions, which enables more flexible responding to stress and psychological symptoms.

\section{Disclosure}

The authors report no conflicts of interest in this work.

\section{References}

1. Paus T, Keshavan M, Giedd, JN. Why do many psychiatric disorders emerge during adolescence? Nat Rev Neurosci. 2008;9(12): 947-957.

2. Kessler RC, Berglund P, Demler O, Jin R, Merikangas KR, Walters EE. Lifetime prevalence and age-of-onset distributions of DSM-IV disorders in the National Comorbidity Survey Replication. Arch Gen Psychiatry. 2005;62(6):593-602.

3. Giedd JN, Blumenthal J, Jeffries NO, et al. Brain development during childhood and adolescence: A longitudinal MRI study. Nat Neurosci. 1999;2(10):861-863.

4. Spear LP. The adolescent brain and age-related behavioral manifestations. Neurosci Biobehav Rev. 2000;24(4):417-463.

5. Lazarus RS, Folkman S. Stress, Appraisal, and Coping. New York, NY: Springer; 1984.

6. Kabat-Zinn J. Wherever you Go, There you are. New York, NY: Hyperion; 1994.

7. Perry-Parrish CK, Sibinga EMS. Mindfulness meditation for children. In: Anbar RD, editor. Functional Symptoms in Pediatric Disease: A Clinical Guide. New York, NY: Springer; 2014:343-352.

8. O'Brien KM, Larson CM, Murrell AR. Third-wave behavior therapies for children and adolescents: Progress, challenges, and future directions. In: Greco LA, Hayes SC, editors. Acceptance and Mindfulness Treatments for Children and Adolescents: A Practitioner's Guide. Oakland, CA: New Harbinger Publications; 2004:15-35.

9. Kabat-Zinn J. Full Catastrophe Living: Using the Wisdom of your Body and Mind to Face Stress, Pain, and Illness. New York, NY: Bantam Books; 1990.
10. Kabat-Zinn J. An outpatient program in behavioral medicine for chronic pain patients based on the practice of mindfulness meditation: Theoretical considerations and preliminary results. Gen Hosp Psychiatry. 1982;4(1):33-47.

11. Kabat-Zinn J, Lipworth L, Burney R. The clinical use of mindfulness meditation for the self-regulation of chronic pain. J Behav Med. 1985;8(2):163-190.

12. Baer RA. Mindfulness training as a clinical intervention: A conceptual and empirical review. Clin Psychol. 2003;10(2):125-143.

13. Segal ZV, Williams JG, Teasdale JD. Mindfulness-based Cognitive Therapy for Depression: A New Approach to Preventing Relapse. New York, NY: Guilford Press; 2002.

14. Linehan MM. Cognitive-behavioral Treatment of Borderline Personality Disorder. New York, NY: Guilford Press; 1993.

15. Hayes SC, Strosahl KD. A Practical Guide to Acceptance and Commitment Therapy. New York, NY: Springer Science + Business Media; 2004.

16. Witkiewitz K, Marlatt GA, Walker D. Mindfulness-based relapse prevention for alcohol and substance use disorders. J Cogn Psychother. 2005;19(3):211-228.

17. Hayes SC, Wilson KG. Acceptance and commitment therapy: Altering the verbal support for experiential avoidance. Behav Anal. 1994;17(2):289-303.

18. Teasdale JD, Segal Z, Williams JG. How does cognitive therapy prevent depressive relapse and why should attentional control (mindfulness) training help? Behav Res Ther. 1995;33(1):25-39.

19. Teasdale JD, Segal Z, Williams JG, Ridgeway VA, Soulsby JM, Lau MA. Prevention of relapse/recurrence in major depression by mindfulness-based cognitive therapy. J Consult Clin Psychol. 2000;68(4): 615-623.

20. Burke CA. Mindfulness-based approaches with children and adolescents: A preliminary review of current research in an emergent field. $J$ Child Fam Stud. 2010;10:133-144.

21. Grant K, Compas B, Thurm A, et al. Stressors and child and adolescent psychopathology: Evidence of moderating and mediating effects. Clin Psychol Rev. 2006;26(3):257-283.

22. Lambert S, Copeland-Linder N, Ialongo N. Longitudinal associations between community violence exposure and suicidality. J Adolesc Health. 2008;43(4):380-386.

23. Broderick PC, Jennings PA. Mindfulness for adolescents: A promising approach to supporting emotion regulation and preventing risky behavior. In: Malti T, editor. Adolescent Emotions: Development, Morality, and Adaptation. San Francisco, CA: Jossey-Bass; 2013:111-126.

24. Garcia Coll C, Lamberty G, Jenkins R, et al. An integrative model for the study of developmental competencies in minority children. Child Dev. 1996;67(5):1891-1914.

25. Perry-Parrish C, Copeland-Linder N, Webb L, Sibinga E. Mindfulnessbased therapies. In: Breland-Noble A, Al-Mateen C, Singh N, editors. Handbook of Mental Health in African American Youth. 1st ed. New York, NY: Springer; 2016:91-105.

26. Kilmer R, Cowen E, Wyman P, Work W, Magnus K. Differences in stressors experienced by urban African American, White, and Hispanic children. J Community Psychol. 1998;26(5):415-428.

27. Evans LD, Kouros C, Frankel SA, et al. Longitudinal relations between stress and depressive symptoms in youth: Coping as a mediator. J Abnorm Child Psychol. 2015;43(2):355-368.

28. Nolen-Hoeksema $\mathrm{S}$. The role of rumination in depressive disorders and mixed anxiety/depressive symptoms. J Abnorm Psychol. 2000; 109(3):504-511.

29. Jimenez SS, Niles BL, Park CL. A mindfulness model of affect regulation and depressive symptoms: Positive emotions, mood regulation expectancies, and self-acceptance as regulatory mechanisms. Pers Individ Dif. 2010;49(6):645-650.

30. Frick PJ, Morris AS. Temperament and developmental pathways to conduct problems. J Clin Child Adolesc Psychol. 2004;33(1):54-68. 
31. Zeman J, Cassano M, Perry-Parrish C, Stegall S. Emotion regulation in children and adolescents. J Dev Behav Pediatr. 2006;27:155-168.

32. Perry-Parrish C, Zeman J. Relations among sadness regulation, peer acceptance, and social functioning in early adolescence: The role of gender. Soc Dev. 2011;20:135-153.

33. Perry-Parrish C, Webb L, Zeman J, et al. Anger regulation and social acceptance in early adolescence: Associations with gender and ethnicity. $J$ Early Adolesc. 2015.

34. Perry-Parrish C, Waasdorp T, Bradshaw C. Peer nominations of emotional expressivity among urban children: Social and psychological correlates. Soc Dev. 2011;21(1):88-108.

35. Breslin FC, Zack M, McMain S. An information-processing analysis of mindfulness: Implications for relapse prevention in the treatment of substance abuse. Clin Psychol. 2002;9(3):275-299.

36. Lynch TR, Chapman AL, Rosenthal MZ, Kuo JR, Linehan MM. Mechanisms of change in dialectical behavior therapy: Theoretical and empirical observations. J Clin Psychol. 2006;62(4):459-480.

37. Kavanagh DJ, Andrade J, May J. Beating the urge: Implications of research into substance-related desires. Addict Behav. 2004;29(7): 1359-1372.

38. Williams M, Teasdale J, Segal Z, Kabat-Zinn J. The Mindful Way through Depression: Freeing Yourself from Chronic Unhappiness. New York, NY: Guilford Press; 2007.

39. Sibinga ES, Kemper KJ. Complementary, holistic, and integrative medicine: Meditation practices for pediatric health. Pediatr Rev. 2010;31(12):91-103.

40. Meiklejohn J, Phillips C, Freedman ML, et al. Integrating mindfulness training into K-12 education: Fostering the resilience of teachers and students. Mindfulness. 2012;3(4):291-307.

41. Zoogman S, Goldberg SB, Hoyt WT, Miller L. Mindfulness interventions with youth: A meta-analysis. Mindfulness. 2014;6(2):290-302.

42. Sibinga EMS, Perry-Parrish C, Thorpe K, Mika M, Ellen JM. A small mixed-method RCT of mindfulness instruction for urban youth. Explore. 2014;10(3):180-186.

43. Sibinga ES, Perry-Parrish C, Chung S, Johnson SB, Smith M, Ellen JM. School-based mindfulness instruction for urban male youth: A small randomized controlled trial. Prev Med. 2013;57(6):799-801.

44. Sibinga ES, Kerrigan D, Stewart M, Johnson K, Magyari T, Ellen JM. Mindfulness-based stress reduction for urban youth. J Altern Complement Med. 2011;17(3):213-218.

45. Tan LB. A critical review of adolescent mindfulness-based programmes. Clin Child Psychol Psychiatry. 2016;21(2):193-207.
46. Barnes VA, Treiber FA, Johnson MH. Impact of transcendental meditation on ambulatory blood pressure in African-American adolescents. Am J Hypertens. 2004;17(4):366-369.

47. Quach D, Jastrowski Mano KE, Alexander K. A randomized controlled trial examining the effect of mindfulness meditation on working memory capacity in adolescents. J Adolesc Health. 2016;58(5):489-496.

48. Atkinson MJ, Wade TD. Mindfulness-based prevention for eating disorders: A school-based cluster randomized controlled study. Int J Eat Disord. 2015;48(7):1024-1037.

49. Sibinga EM, Stewart M, Magyari T, Welsh CK, Hutton N, Ellen JM. Mindfulness-based stress reduction for HIV-infected youth: A pilot study. Explore. 2008;4(1):36-37.

50. Kerrigan D, Johnson K, Stewart M, et al. Perceptions, experiences, and shifts in perspective occurring among urban youth participating in a mindfulness-based stress reduction program. Complement Ther Clin Pract. 2011;17(2):96-101.

51. Webb L, Ghazarian S, Perry-Parrish C, Ellen J, Sibinga E. Mindfulness instruction for urban, HIV-positive youth: A small randomized controlled trial. J Early Adolesc. Epub 2015 Oct 28.

52. Sibinga EM, Webb L, Ghazarian SR, Ellen JM. School-based mindfulness instruction: an RCT. Pediatrics. 2016;137(1):1-8.

53. Biegel GM, Brown KW, Shapiro SL, Schubert CM. Mindfulness-based stress reduction for the treatment of adolescent psychiatric outpatients: A randomized clinical trial. J Consult Clin Psychol. 2009;77(5):855-866.

54. Bootzin RR, Stevens SJ. Adolescents, substance abuse, and the treatment of insomnia and daytime sleepiness. Clin Psychol Rev. 2005;25(5):629-644.

55. Lee J, Semple RJ, Rosa D, Miller L. Mindfulness-based cognitive therapy for children: Results of a pilot study. J Cogn Psychother. 2008;22(1):15-28.

56. Semple RJ, Lee J, Rosa D, Miller LF. A randomized trial of mindfulnessbased cognitive therapy for children: Promoting mindful attention to enhance social-emotional resiliency in children. J Child Fam Stud. 2010;19(2):218-229.

57. Bögels S, Hoogstad B, van Dun L, de Schutter S, Restifo K. Mindfulness training for adolescents with externalizing disorders and their parents. Behav Cogn Psychoth. 2008;36:193-209.

58. Haydicky J, Shecter C, Wiener J, Ducharme JM. Evaluation of MBCT for adolescents with ADHD and their parents: Impact on individual and family functioning. J Child Fam Stud. 2015;24:76-94.

59. Ames CS, Richardson J, Payne S, Smith P, Leigh E. Innovations in practice: Mindfulness-based cognitive therapy for depression in adolescents. Child Adolesc Ment Health. 2014;19:74-78.
Adolescent Health, Medicine and Therapeutics

Publish your work in this journal

Adolescent Health, Medicine and Therapeutics is an international, peer-reviewed, open access journal focusing on health, pathology, and treatment issues specific to the adolescent age group. All aspects of health maintenance, preventative measures and disease treatment interventions are addressed within the journal and practitioners from all disciplines are

\section{Dovepress}

invited to submit their work as well as healthcare researchers and patient support groups. This journal is included in PubMed. The manuscript management system is completely online and includes a very quick and fair peer-review system. Visit http://www.dovepress.com/testimonials. php to read real quotes from published authors. 